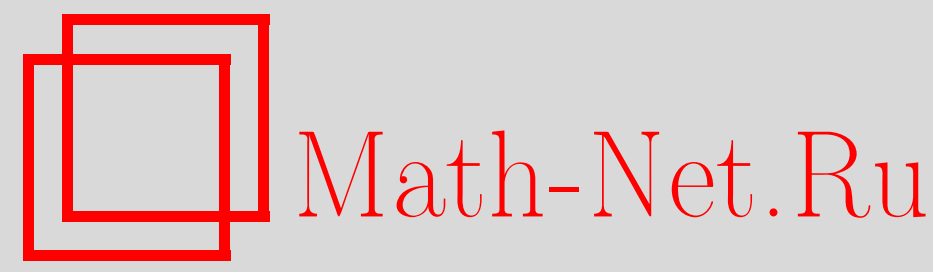

В. Э. Кордонский, О бирациональной классификации действий алгебраических групп, Изв. РАН. Сер. матем., 2001, том 65, выпуск 1, 61-76

DOI: https://doi.org/10.4213/im319

Использование Общероссийского математического портала Math-Net.Ru подразумевает, что вы прочитали и согласны с пользовательским соглашением

http://www . mathnet.ru/rus/agreement

Параметры загрузки:

IP: 34.239 .49 .27

26 апреля 2023 г., $16: 12: 05$ 
УДК 512.745

\title{
В.Э. Кордонский
}

\section{О бирациональной классификации действий алгебраических групп}

\begin{abstract}
В настоящей работе показывается, что в ряде случаев бирациональная классификация некоторых действий произвольной алгебраической группы может быть сведена к бирациональной классификации локально свободных действий редуктивных групп. Доказывается существование обычных и относительных сечений для некоторых действий.

Библиография: 18 наименований.
\end{abstract}

\section{§1. Введение}

Настоящая работа посвящена бирациональной классификации действий линейных алгебраических групп над алгебраически замкнутым полем $k$ характеристики нуль и некоторым смежным вопросам бирациональной теории инвариантов. Все необходимые факты и определения можно найти в [2], [3], [7].

ОПРЕДЕЛЕНИЕ 1. 1) Назовем действия $G: X_{1}$ и $G: X_{2}$ бирационально изоморфными , если существует $G$-эквивариантное бирациональное отображение $f$ : $X_{1} \rightarrow X_{2}$.

2) Действие группы $G$ на многообразии $X$ называется действием со стабилизатором общего положения, если существует такое плотное открытое подмногообразие $W \subset X$, что для всякого $x \in W$ стабилизатор $G_{x}$ сопряжен некоторой фиксированной подгруппе $H \subset G$. При этом $H$ называется стабилизатором общего положения действия $G: X$.

Везде в данной работе, кроме $\S 3$ и п. 4.1, мы ограничимся рассмотрением рациональных действий алгебраической группы $G$ на алгебраическом многообразии $X$, для которых группа $G$ действует транзитивно на множестве неприводимых компонент $X$. В этом случае определено поле инвариантов $K=k(X)^{G}$.

ОПРЕДЕЛЕНИЕ 2. Произвольное многообразие $Y$, поле рациональных функций которого равно $K$, называется рациональнылм фактором действия $G$ на $X$, а естественное отображение $\pi: X \rightarrow Y$ - отображсением факторизации.

Подмножество $W \subset X$ называется густым, если $W$ содержит плотное открытое по Зарисскому подмножество многообразия $X$.

Действие $G: X$ называется локально свободным, если существует такое густое подмногообразие $W \subset X$, что стабилизатор $G_{w}$ любой точки $w \in W$ тривиален.

Одними из основных понятий и объектов изучения бирациональной теории инвариантов являются понятия квазисечения и сечения действия.

Работа выполнена при финансовой поддержке грантов INTAS-OPEN-97-1570 и CRDF RM1-206.

(C) В.Э. Кордонский, 2001 
ОПРЕДЕЛЕНИЕ 3. Неприводимое подмногообразие $S \subset X$ называется сечением (соответственно квазисечением) действия $\alpha$, если сушествует такое инвариантное густое подмножество $X_{0} \subset X$, что любая орбита индуцированного действия $G: X_{0}$ пересекает $S$ ровно в одной точке (соответственно по непустому конечному множеству).

Сечение существует далеко не всегда. В то же время любое действие допускает квазисечение (см., например, [3]).

В данной работе большую роль играют различные обобщения (предложение 4 и теоремы 3 и 12) известной теоремы Розенлихта.

ТЕОРема РОЗЕНЛИХТА [14]. Пусть $V$ - произвольная связная разрешимая группа. Тогда любое действие группь $V$ допускает сечение.

В дальнейшем теорема Розенлихта будет использоваться лишь для унипотентных групп.

Известно, что для локально свободных действий их бирациональная классификация сводится к вычислению некоторых когомологий Галуа (см. [3]). Аналогичный результат может быть получен для действий с произвольным стабилизатором общего положения (см. [3]).

В данной работе рассматривается бирациональная классификация произвольных действий и показывается, что ее также можно свести к вычислению когомологий Галуа, а точнее, когомологий Галуа некоторых редуктивных групп.

В $\S 2$ мы покажем, как каждому действию $\alpha$ со стабилизатором общего положения можно канонически сопоставить локально свободное действие $\Psi(\alpha)$ некоторой редуктивной группы и тем же полем инвариантов. При этом действия $\alpha$ и $\beta$ с одинаковыми стабилизаторами общего положения бирационально изоморфны тогда и только тогда, когда действия $\Psi(\alpha)$ и $\Psi(\beta)$ бирационально изоморфны. Следовательно, можно рассмотреть отображение $\Psi$ на классах бирационально изоморфных действий. Мы покажем (теорема 1$)$, что $\Psi$ является биекцией.

Существование такого отображения $\Psi$ следует из [3] и [17] (см. теорему 5 и п. 4.1 настоящей работы).

В $\S 3$ приводятся необходимые для дальнейших исследований основные результаты теории однородных пространств над алгебраически незамкнутыми полями.

В $\S 4$ строится обобщение отображения $\Psi$ на случай произвольных действий (теорема 7). Это отображение в общем случае зависит от выбора "начального" действия $\alpha_{0}$. Построение этого отображения $\Psi$ есть не что иное, как объединение результатов [3], [17], [7] (см. $\S 3$ и п. 4.1 настояшей работы). В частном случае, когда стабилизатор квазисечения определен над полем инвариантов, отображение $\Psi$ не зависит от начального действия (теорема 5).

Стабилизатор сечения определен над полем инвариантов, поэтому в связи с теоремой 5 для бирациональной теории действий представляют интерес результаты о сушествовании сечений. Некоторые из них приводятся в п. 2.4. Эти теоремы могут быть получены из результатов теории однородных пространств над алгебраически незамкнутыми полями при помощи результатов п. 4.1. Доказательству теоремы о существовании сечения для действия специальных групп с унипотентными стабилизаторами (теоремы 12) посвяшается $\S 6$. 
В $\S 7$ формулируется и доказывается одно достаточное условие сушествования относительного сечения (определение см. в $§ 3$ ). При этом доказательство сушественно опирается на результаты $\S 2,6$.

\section{§2. Случай действия, имеющего стабилизатор общего положения}

2.1. Относительное сечение. Пусть задано действие группы $G$ на многообразии $X$ и $P \subset G$ - некоторая подгруппа.

ОПРЕДЕЛЕНИЕ 4 [4]. Несмешанное подмногообразие $S \subset X$ называется относительным сечением или $P$-сечением, если выполнены следуюшие три условия:

1) $\overline{G S}=X$

2) $P S \subset S$;

3 ) существует такое густое подмногообразие $S_{0} \subset S$, что $g S_{0} \cap S_{0} \neq \varnothing$ влечет $g \in P$.

Как легко видеть, поля инвариантов действий $G: X$ и $P: S$ естественно изоморфны.

ПРЕДЛОЖЕНИЕ 1 (см. [3] и [12]). Многообразие X с действием на нем группь $G$ однозначно с точностью до бирачионального изоморфизма восстанавливается по $P$-сечению $S$ с действием на нем подгруппь $P \subset G$.

Пусть теперь некоторая подгруппа $P \subset G$ действует на некотором многообразии $S$. Положим $X$ равным рациональному фактору действия $P: G \times S$, задаваемого формулой

$$
p(g, s)=\left(g p, p^{-1} s\right) .
$$

Рассмотрим действие $G: X$, индуцированное действием $G: G \times S$ на первом множителе левыми умножениями.

ПРЕДЛОЖЕНИЕ 2 (см. [12]). Построенное выше действие $G: X$ допускает такое $P$-сечение $S^{\prime}$, что действия $P: S$ и $P: S^{\prime}$ изоморфны.

2.2. Сведе́ние к случаю локально свободного действия. Пусть $\alpha$ - действие группы $G$ на многообразии $X$ со стабилизатором общего положения, равным $H$. Рассмотрим объединение $X_{m}^{H}$ неприводимых компонент максимальной размерности многообразия $X^{H}$, состоящего из неподвижных относительно $H$ точек $X$. Легко видеть, что $X_{m}^{H}$ является $N_{G}(H)$-сечением.

Более того, $N_{G}(H)$ на $X_{m}^{H}$ действует с ядром $H$, т. е. действие группы $N_{G}(H) / H$ на $X_{m}^{H}$ является локально свободным действием.

Обозначим последнее действие через $\psi_{1}(\alpha)$. Отображение $\psi_{1}$ сопоставляет действиям группы $G$ со стабилизатором общего положения $H$ локально свободные действия групшы $N_{G}(H) / H$.

Легко видеть, что из предложений 1 и 2 следует

Лемма 1. $\psi_{1}$ - биекиия.

2.3. Существование инвариантного сечения. Рассмотрим произвольное разложение в полупрямое произведение $G=M \wedge V$ группы $G$ (группа $V$ является нормальной подгруппой группы $G$ ).

Пусть $\alpha$ - действие группы $G$ на многообразии $X$. 
ПРЕДЛОЖЕНИЕ 3. Действие $V: X$ допускает $M$-инвариантное квазисечение.

ДокаЗАТЕЛЬСтво. Пусть $S$ - квазисечение действия $G: X$. Рассмотрим множество

$$
S^{\prime}=\{x \in X \mid x=m \cdot s, m \in M, s \in S\} \subset X .
$$

Его плотное подмножество является $M$-инвариантным квазисечением действия $V: X$.

Пусть $Y$ - неприводимое многообразие. Рассмотрим многообразие $Y \times k^{m}$, где естественно определена сумма точек $\left(y, v_{1}\right)$ и $\left(y, v_{2}\right)$ для любых $y \in Y$ и $v_{1}, v_{2} \in$ $k^{m}$. Пусть замкнутое подмногообразие $S \subset Y \times k^{m}$ такое, что ограничение на $S$ проекции $Y \times k^{m} \rightarrow Y$ задает неразветвленное $n$-листное накрытие $Y$. Определим множество $S^{\prime} \subset Y \times k^{m}$ как множество точек вида

$$
\left(y, \frac{1}{n} \sum_{\{v \mid(y, v) \in S\}} v\right)
$$

Лемма 2. Можнн выбрать густое подмногообразие $Y_{0} \subset Y$ таким образом, что множество $S^{\prime} \cap\left(Y_{0} \times k^{m}\right)$ будет являться подмногообразием многообразия $Y_{0} \times k^{m}$ и сечением векторного расслоения $Y_{0} \times k^{m} \rightarrow Y_{0}$.

ДокАЗАТЕЛЬСтво. Выберем некоторое координатное разложение $k^{m}=$ $\bigoplus_{i=1}^{m} k_{i}$ так, чтобы образы $S$ при проекциях $\pi_{i}: Y \times k^{m} \rightarrow Y \times k_{i}$ являлись $n$-листными накрытиями многообразия $Y$. Так как $\operatorname{codim}_{Y \times k_{i}} \pi_{i}(S)=1$, то для некоторого непустого открытого афффинного подмногообразия $Y_{i} \subset Y$ выполняются следуюшие свойства:

1) подмногообразие $S_{i}=\left(\pi_{i}(S) \cap Y_{i} \times k_{i}\right) \subset\left(Y_{i} \times k_{i}\right)$ является неразветвленным накрытием многообразия $Y_{i}$;

2 ) многообразие $S_{i}$ задается одним уравнением

$$
x_{i}^{n}+a_{i, n-1} x_{i}^{n-1}+\cdots+a_{n, 1} x_{i}+a_{i, 0}=0,
$$

где $a_{i, j}$ - регулярные функции на $Y_{i}$ и $x_{i}$ - координатная функция на $k_{i}$.

Покажем, как можно добиться выполнения условия 2). Пусть мы выбрали многообразие $Y_{i}$, удовлетворяющее условию 1). Рассмотрим идеал $I=I\left(\pi_{i}(S)\right) \subset$ $k\left(Y_{i}\right)\left[x_{i}\right]$, состоящий из многочленов, обнуляющихся на $\pi_{i}(S)$. Это главный идеал. Обозначим его образующую через $f$. Переходя к непустому открытому подмножеству $Y_{i}$, можно считать, что $f \in k\left[Y_{i}\right]\left[x_{i}\right]$ и коэффициент многочлена $f$ при старшем члене по переменной $x_{i}$ равен 1 . Так как поле $k$ имеет нулевую характеристику и идеал $I$ радикален, то многочлен $f$ и его производная $f^{\prime}$ по переменной $x_{i}$ взаимно просты в $k\left(Y_{i}\right)\left[x_{i}\right]$. Следовательно, существуют $g, h$ в кольце $k\left(Y_{i}\right)\left[x_{i}\right]$ такие, что $g f+h f^{\prime}=1$. Переходя к непустому открытому подмножеству $Y_{i}$, можно считать, что $g, h \in k\left[Y_{i}\right]\left[x_{i}\right]$. Для произвольной точки $y \in Y_{i}$ многочлен $f(y) \in k\left[x_{i}\right]$ имеет ровно $n$ корней. С другой стороны, $f(y)$ не имеет кратных корней, так как $g(y) f(y)+h(y) f^{\prime}(y)=1 \in k\left[x_{i}\right]$ и, следовательно, многочлен $f(y)$ взаимно прост со своей производной. Значит, степень $f(y)$ равна $n$. Следовательно, степень многочлена $f$ по переменной $x_{i}$ также равна $n$. 
Положим теперь $Y_{0}=\bigcap_{i} Y_{i}$. По теореме Виета множество $\left(S^{\prime} \cap\left(Y_{0} \times k^{m}\right)\right) \subset$ $Y_{0} \times k^{m}$ задается уравнениями

$$
x_{i}=-\frac{1}{n} a_{i, n-1} .
$$

Мы показали, что $S^{\prime} \cap\left(Y_{0} \times k^{m}\right)$ - замкнутое подмногообразие многообразия $Y_{0} \times k^{m}$. То, что многообразие $S^{\prime} \cap\left(Y_{0} \times k^{m}\right)$ является сечением расслоения $Y_{0} \times k^{m} \rightarrow Y_{0}$, следует из построения множества $S^{\prime}$.

ПРЕДЛОЖЕНИЕ 4. Если $V$ - унипотентная группа, то действие $V: X$ допускает $M$-инвариантное сечение.

ДокАЗАТЕльство. Пусть вначале $V$ - векторная группа.

По теореме Розенлихта действие $V: X$ допускает сечение и, следовательно, определено $V$-эквивариантное отображение $f: Y \times V \rightarrow X$, где $Y$ - рациональный фактор действия $V: X$.

Пусть размерность пространства $V$ равна $n$, а размерность стабилизаторов точек из некоторого плотного открытого подмножества многообразия $Y$ равна $n-m$. Выберем в $V$ подпространство $U$ размерности $m$, трансверсальное к стабилизаторам точек из густого подмножества многообразия $Y$. Так как свойство иметь ненульмерное пересечение с некоторым подпространством $V$ выделяет замкнутое подмногообразие в грассманиане $\operatorname{Gr}(V, n-m)$, то в качестве пространства $U$ можно взять любое $m$-мерное пространство, трансверсальное к произвольному стабилизатору точки из $X$. По построению отображение $\left.f\right|_{Y \times U}: Y \times U \rightarrow X$ является бирациональным изоморфизмом.

Таким образом, действие $V: X$ бирационально изоморфно действию $V: Y \times k^{m}$ для $Y$, равного $X / V$, и некоторого натурального $m$, где действие $V: Y$ тривиально, а действие $V:\{y\} \times k^{m}$ зависит от точки $y \in Y$.

По предложению 3 выберем $M$-инвариантное квазисечение $S \subset X$ для действия $V: Y \times k^{m}$. Переходя к замыканию в $Y \times k^{m}$, можно считать, что $S$ замкнуто в $Y \times k^{m}$, а переходя к непустому открытому подмножеству в $Y$, можно считать, что отображение факторизации $Y \times k^{m} \rightarrow Y$ при ограничении на $S$ является неразветвленным накрытием.

Вновь переходя к непустому открытому подмногообразию $Y$, получаем, что по лемме 2 определено подмногообразие $S^{\prime}$, которое является сечением действия $V$ : $Y \times k^{m}$.

Для доказательства предложения для векторной группы $V$ остается проверить $M$-инвариантность сечения $S^{\prime}$.

Пусть $s^{\prime}$ - произвольная точка из $S^{\prime}$. Тогда $s^{\prime}=v_{i} s_{i}$, где $v_{i} \in V$ и $s_{i} \in S$-точки, лежащие на той же $V$-орбите, что и $s^{\prime}$. Для любого элемента $m \in M$ получаем

$$
m s^{\prime}=m v_{i} s_{i}=\left(m v_{i} m^{-1}\right) m s_{i} .
$$

Точки $m s_{i}$ принадлежат многообразию $S$ в силу его $M$-инвариантности.

Так как группа $V$ абелева, то по построению многообразия $S^{\prime}$ имеем

$$
\sum_{i=1}^{n} v_{i}=0 \in V .
$$


Для доказательства того, что $m s^{\prime} \in S^{\prime}$, достаточно проверить, что

$$
\sum_{i=1}^{n}\left(m v_{i} m^{-1}\right)=0 \in V
$$

но это равенство имеет место в силу предыдущего равенства и линейности действия подгруппы Леви на унипотентном радикале.

В случае абелевого унипотентного радикала предложение доказано.

Доказательство для произвольной унипотентной группы $V$ получается индукцией по размерности $V$. База индукции уже доказана. Рассмотрим центр $Z$ группы $V$. По предположению индукции действие группы $M \wedge(V / Z)=(M \nless V) / Z$ на многообразии $X / Z$ допускает $M$-инвариантное $V / Z$-сечение $S$. Рассмотрим $S^{\prime}-$ полный прообраз $S$ при отображении $X \rightarrow X / Z$. Действие $M \wedge Z: S^{\prime}$ по предположению индукции допускает $M$-инвариантное $Z$-сечение $S^{\prime \prime}$. Подмногообразие $S^{\prime \prime} \subset X$ является $M$-инвариантным $V$-сечением, так как $Z S^{\prime \prime}$ густо в $S^{\prime}$, а $V S^{\prime}$ густо в $X$.

2.4. Сведе́ние к случаю редуктивной группы. Рассмотрим разложение Леви $G=M \wedge V$ группы $G$.

Пусть задано некоторое локально свободное действие $M: Y$. Определим локально свободное действие $G: V \times Y$ по формуле

$$
g \cdot(v, y)=\left(m u v m^{-1}, m y\right)=\left(g v m^{-1}, m y\right)
$$

где $m u=g \in G, m \in M, u \in V$ и $(v, y) \in V \times Y$.

ПРЕДЛОЖЕНИЕ 5. Любое локально свободное действие группь $G: X$ может быть построено описанным выше способом по некоторому локально свободному действию $M: Y$.

ДокАЗАтЕльство. Пусть $G: X$ - некоторое локально свободное действие. По предложению 4 возьмем $M$-инвариантное сечение $Y$ действия $V: X$. По действию $M: Y$ построим действие $G: V \times Y$. Легко видеть, что отображение

$$
(v, y) \mapsto v \cdot y
$$

задает бирациональный изоморфизм между действиями $G: V \times Y$ и $G: X$.

Лемма 3. Действия $M: Y$ и $M:(V \times Y) / V$ бирачионально изоморфнь.

ДоказАТЕЛьСтво. Определим отображение $Y \rightarrow(V \times Y) / V$ по формуле $y \rightarrow$ $(e, y) V$. Это отображение является бирациональным и $M$-эквивариантным.

Пусть $\alpha$ - локально свободное действие группы $G$ на многообразии $X$. Тогда определено естественное действие группы $M$ на $X / V$, которое, как легко видеть, также является локально свободным.

Обозначим последнее действие через $\psi_{2}(\alpha)$. Отображение $\psi_{2}$ сопоставляет локально свободным действиям группы $G$ локально свободные действия группы $M$. Из предложения 5 и леммы 3 получаем следующую лемму. 
Лемма 4. $\psi_{2}-$ биекиия.

Итак, мы научились по действию $\alpha$ группы $G$ со стабилизатором общего положения $H$ строить локально свободное действие $\Psi(\alpha)=\psi_{2}\left(\psi_{1}(\alpha)\right)$ подгруппы Леви $F$ группы $N_{G}(H) / H$.

Из лемм 1 и 4 получаем следующую теорему.

Teоpema 1. $\Psi$ - биекuия.

Легко доказываются следуюшие два свойства отображение $\Psi$ :

1) $\alpha$ и $\Psi(\alpha)$ имеют одно и то же поле инвариантов;

$2)$ пусть $\alpha$ и $\beta$ - действия $G: X$ и $G: Y$ со стабилизаторами общего положения $H$ и $\Psi(\alpha)$ и $\Psi(\beta)$ - локально свободные действия $F: X^{\prime}$ и $F: Y^{\prime}$; если задано $G$-эквивариантное доминантное отображение $\iota: X \rightarrow Y$, то канонически определено $F$-эквивариантное доминантное отображение $\varphi_{*}(\iota): X^{\prime} \rightarrow Y^{\prime}$, сопоставление $\iota \rightarrow \varphi_{*}(\iota)$ функториально, т. е. если $\iota_{1} \circ \iota_{2}=\iota$, то $\varphi_{*}\left(\iota_{1}\right) \circ \varphi_{*}\left(\iota_{2}\right)=\varphi_{*}(\iota)$.

\section{§3. Действия над алгебраически незамкнутыми полями}

Обозначим через $K \supset k$ произвольное поле, а через $\bar{K}$ его алгебраическое замыкание.

Алгебраическое многообразие, определенное над $K$, мы будем отождествлять с множеством его $\bar{K}$-точек.

3.1. Однородные пространства и когомологии Галуа. Пусть $G$ - алгебраическая группа над $K$ и $Q, Q^{\prime}$ - однородные пространства группы $G$, определенные над $K$. Многообразие $Q^{\prime}$ называется $\bar{K} / K$-формой однородного пространства $Q$, если $Q$ как однородное пространство группы $G$ изоморфно $Q^{\prime}$ над $\bar{K}$. Классы таких форм по отношению эквивалентности, определенному $K$-изоморфизмами, образуют множество $E(\bar{K} / K, Q)$.

Обозначим через $\operatorname{Aut}_{G}(Q)$ группу $G$-эквивариантных автоморфизмов однородного пространства $Q$.

Tеорема 2 [7]. Существует каноническая биекция между множеством $E(\bar{K} / K, Q)$ и множеством когомологий Галуа $H^{1}\left(K, \operatorname{Aut}_{G}(Q)\right)$.

Пусть $A$ - некоторая группа, определенная над $K$. Пусть $B$ - ее нормальная подгруппа, также определенная над $K$. Рассмотрим естественное отображение $\psi: H^{1}(K, A) \rightarrow H^{1}(K, A / B)$.

Теорема 3 [17], [6]. Если группа В унипотентна, то $\psi$ - биекция.

Обозначим через $R_{u} A$ унипотентный радикал группы $A$.

Положим $F=\operatorname{Aut}_{G}(Q) / R_{u} \operatorname{Aut}_{G}(Q)$. Тогда по теореме 3 получаем следующую теорему.

Теорема 4. Существует каноническая биекиия между множествами $E(\bar{K} / K, Q) u H^{1}(K, F)$.

Пусть $H \subset G$ - стабилизатор некоторой точки $q \in Q$. Тогда существует определенный над $\bar{K}$ изоморфизм групп $N_{G}(H) / H$ и $\operatorname{Aut}_{G}(Q)$. 
Теорема 5. Пусть $H \subset G$ определена над $K$. Тогда существует каноническая биекиия между множествами $E(\bar{K} / K, Q)$ и $H^{1}\left(K,\left(N_{G}(H) / H\right) /\right.$ $\left.R_{u}\left(N_{G}(H) / H\right)\right)$.

ДокаЗАТЕЛЬСтво. Пусть $Q^{\prime}-\bar{K} / K$-форма однородного пространства $Q$. Многообразие $\left(Q^{\prime}\right)^{H}$ является главным однородным пространством группы $N_{G}(H) / H$, определенным над $K$.

Обратно, пусть $Z$ - главное однородное пространство группы $N_{G}(H) / H$, определенное над $K$. Многообразие $G \times{ }_{N_{G}(H)} Z$ является однородным пространством групшы $G$, определенным над полем $K$. Кроме того, $G \times_{N_{G}(H)} Z-\bar{K} / K$-форма однородного пространства $Q$. Поэтому множество $E(\bar{K} / K, Q)$ канонически биективно множеству $E\left(\bar{K} / K, N_{G}(H) / H\right)$.

Применение теорем 4 и 3 завершает доказательство теоремы.

\section{2. Редуктивные и параболические подгруппы.}

ТЕОРЕМА 6. Всякая редуктивная или параболическая подгруппа группь $G(\bar{K})$ сопряжена в $G(\bar{K})$ некоторой подгруппе, определенной над $k$.

ДокАЗАТЕльСтво. Эта теорема следует из того, что классификация редуктивных и параболических подгрупп данной группы с точностью до сопряженности не зависит от алгебраически замкнутого поля [8].

\section{§4. Случай действия с произвольными стабилизаторами}

4.1. Действия и однородные пространства. Пусть $\alpha$ - действие алгебраической группы $G$ на алгебраическом многообразии $X, K$ - поле инвариантов, $Y$ рациональный фактор, $\pi$ - отображение факторизации.

В этом параграфе нам придется несколько изменить определение квазисечения. А именно, квазисечением действия $\alpha$ мы будем называть отображение $\sigma$ некоторого неприводимого алгебраического многообразия $Z$ в многообразие $X$, для которого отображение $\rho=\pi \circ \sigma: Z \rightarrow Y$ является рациональным накрытием. Сечением действия $\alpha$ называется такое отображение $\sigma: Y \rightarrow X$, что $\sigma \circ \pi=\operatorname{id}_{Y}$.

Несколько квазисечений по новому определению могут задавать одно и то же квазисечение по определению, данному во введении.

Рассмотрим действие $\kappa(\alpha)$ группы $G(\bar{K})$ на многообразии $Q$ квазисечений действия $\alpha$. Известно [3], что $Q$ является однородным пространством групшы $G(\bar{K})$, определенным над $K$. Более того [3], отображение $\kappa$ биективно отображает множество классов бирационально эквивалентных действий группы $G$ с полем инвариантов $K$ во множество однородных пространств группы $G(\bar{K})$, определенных над $K$. Основную идею, используемую при построении отображения $\kappa^{-1}$, см. в [3].

Сечения действия $\alpha$ суть в точности $K$-точки многообразия $Q$ [3]. Поэтому теорему Розенлихта (см. введение к данной работе) можно переформулировать следующим образом.

ТЕОРемА РОЗЕНЛИХТА. Любое однородное пространство связной разрешимой группь имеет рациональную точку. 
4.2. Классификация действий. Рассмотрим многообразие $Q$ квазисечений некоторого действия $\alpha_{0}$ группы $G$. Положим $F=\operatorname{Aut}_{G}(Q) / R_{u} \operatorname{Aut}_{G}(Q)$.

Согласно сказанному в предыдущем пункте, теорему 4 можно переформулировать следуюшим образом.

Теорема 7. Пусть $\alpha_{0}$ - действие группь $G$ с полем инвариантов $K$ и $\mathrm{H}$ стабилизатор квазисечения $q$ действия $\alpha_{0}$. Тогда существует каноническое биективное отображение $\Psi$ множества действий группы $G$ с полем инвариантов $K$ и стабилизатором квазисечения $H$ во множество $H^{1}(K, F)$.

ЗАмечАниЕ 1 . Не произвольная подгруппа $H \subset G(\bar{K})$ может быть реализована как стабилизатор квазисечения некоторого действия группы $G$ с полем инвариантов $K$. Для построения контрпримера достаточно найти такую подгруппу $H \subset G(\bar{K})$, что однородное пространство $G(\bar{K}) / H$ группы $G(\bar{K}) / H$ не может быть определено над $K$. В частности, $H$ не может являться стабилизатором квазисечения в случае, когда $H$ и $H^{\gamma}$ не сопряжены при каком-нибудь $\gamma \in \operatorname{Gal}\left(K^{\prime} / K\right)$ для некоторого $K^{\prime} \supset K$. Рассмотрим группу $G=k \times k$, поля $K^{\prime}=k(x)$, $K=k\left(x^{2}\right)$ и группу $H \subset G\left(K^{\prime}\right) \subset G(\bar{K})$, определяемую как семейство подгрупп $H(x)=\{(a,-a x) \mid a \in k\} \subset G$, параметризованных $x \in k$. Ввиду коммутативности группы $G$ подгруппы $H\left(x_{1}\right)$ и $H\left(x_{2}\right)$ при $x_{1} \neq x_{2}$ не сопряжены. Следовательно, для нетривиального элемента $\gamma \in \operatorname{Gal}\left(K^{\prime} / K\right)$ подгруппа $H^{\gamma}$ не сопряжена $H$. Аналогичный пример можно построить и для редуктивной группы $G^{1}$.

Если подгруппа $H \subset G(\bar{K})$ определена над $K$, то в теореме 7 нет необходимости в выборе "начального действия" $\alpha_{0}$. А именно, согласно сказанному в предыдущем пункте теорему 5 можно переформулировать следуюшим образом.

Tеорема 8. Пусть $K$ - некоторое функциональное поле, подгруппа $H \subset$ $G(\bar{K})$ определена над $K$. Тогда существует каноническое биективное отображение $\Psi$ множества действий группы $G$ с полем инвариантов $K$ и стабилизатором квазисечения $H$ во множество

$$
H^{1}\left(K,\left(N_{G}(H) / H\right) / R_{u}\left(N_{G}(H) / H\right)\right) .
$$

\section{$\S 5$. Существование сечений}

5.1. Случай одномерного фактора. В силу теоремы 8 особый интерес для бирациональной классификации действий представляет вопрос о сушествовании сечений.

Результаты [3] (см. п. 4.1 данной работы) позволяют сформулировать теоремы, касающиеся рациональной теории действий, ранее известные автору только на языке когомологий Галуа (см. [7]).

\footnotetext{
${ }^{1}$ А именно, возьмем группу $G=\mathrm{SL}_{6}$ и поля $K^{\prime}=k(x), K=k\left(x^{2}\right)$. Рассмотрим такую унипотентную подгруппу $H(x)$ группы $G$, что ее касательная алгебра $\mathfrak{h}(x)$ порождена матрицами $E_{25}+E_{56}, E_{13}+x E_{34}, E_{12}+E_{46}, E_{15}, E_{36}$ и $E_{16}$, где $x \in k$ фиксирован и через $E_{i j}$ обозначены матричные единицы. Согласно результатам [5] группы $H(x)$ не изоморфны при различных $x$. Рассмотрим группу $H \subset G\left(K^{\prime}\right) \subset G(\bar{K})$, определяемую как семейство подгрупп $H(x) \subset G$, параметризованных $x \in k$. Легко видеть, что для нетривиального элемента $\gamma \in \operatorname{Gal}\left(K^{\prime} / K\right)$ подгруппа $H^{\gamma}$ не изоморфна, а значит, и не сопряжена $H$, что и требовалось показать. (Данный пример любезно предоставлен автору Е.А. Тевелевым.)
} 
Предположим, что $\operatorname{tr} . \operatorname{deg}_{k} K \leqslant 1$. Тогда $K$ квазиалгебраически замкнуто (см., например, [11]).

ТЕОРема 9. Любое действие связной группь $G$ с полем инвариантов $K$ допускает сечение.

ТЕОРема 10. Пусть $\alpha$ - действие группь $G$ с полем инвариантов $K u$ стабилизатором сечения $H$.

Тогда существует каноническое биективное отобрахение множества действий $\beta$ с полем инвариантов $K$ и стабилизатором квазисечения $Н$ на множество локально свободньх действий группьь $\left(N_{G}(H) / H\right) /\left(N_{G}(H) / H\right)^{0}$ с полем инвариантов $K$.

Таким образом, бирациональная классификация действий групп с одномерньм фактором сводится к бирациональной классификации действий конечных групп с тем же фактором.

Пусть $A-K$-группа и $X$ - ее однородное пространство, определенное над $K$.

Теоремы 9 и 10 являются переформулировками следуюших результатов [7, гл. III, следствия 1 и 3 теоремы 3$]$.

СлЕДСТВИЕ 1. Если tr. $\operatorname{deg}_{k} K \leqslant 1 u H^{1}(K, A)=0$, mo $X$ имеет $K$-точку.

СлЕДСТВИЕ 2. Если $\operatorname{tr} \cdot \operatorname{deg}_{k} K \leqslant 1$, то каноническое отображсение $H^{1}(K, A) \rightarrow H^{1}\left(K, A / A^{0}\right)$ биективно.

\section{2. Специальные группы.}

ОПРЕДЕЛЕНИЕ 5. Алгебраическая группа $G$ называется сnециальной, если выполнено одно из следующих эквивалентных условий:

1) любое локально свободное действие группы $G$ допускает сечение;

2 ) для любого поля алгебраических функций $K$ все локально свободные действия группы $G$ с полем инвариантов $K$ бирационально изоморфны;

3) для любого поля $K \supset k$ выполнено $H^{1}(K, G)=0$.

Эквивалентность 1) и 2) очевидна, эквивалентность 2) и 3) опирается на связь между действиями алгебраических групп и однородными пространствами над алгебраически незамкнутьми полями (см. п. 4.1).

ЗАМЕЧАНИЕ 2. Отметим, что условия:

$\left.1^{\prime}\right)$ любое действие группы $G$ со стабилизатором общего положения $H$ и полем инвариантов $K$ допускает сечение,

$\left.2^{\prime}\right)$ все действия группы $G$ со стабилизатором общего положения $H$ и полем инвариантов $K$ бирационально изоморфны,

не равносильны (см. [12]).

Приведем основной классификационный результат.

Теорема 11 [15]. Группа $G$ специальна тогда и только тогда, когда $G$ связная группа, максимальная полупростая подгруппа которой есть прямое произведение групп типов SL $и$ Sp. 


\section{3. Действия специальных групп с унипотентными стабилизатора-} ми.

Теорема 12. Любое действие специальной группы $G: X$ с унипотентными стабилизаторами точек из некоторого густого подмнодсества $W \subset X$ допускает сечение.

Доказательство этой теоремы может быть получено на основе результатов [7] и [3]. В настоящей работе мы предлагаем геометрическое доказательство, которое буде дано ниже.

\section{§6. Доказательство теоремы 12}

6.1. Правильное вложение произвольной подгруппы в параболическую подгруппу. Пусть $H$ - некоторая алгебраическая подгруппа группы связной алгебраической группы $G$. Тогда по $H$ можно однозначно построить параболическую подгруппу. А именно, положим $N^{0}(H)=H$ и рассмотрим $U^{1}(H)=R_{u} H-$ унипотентный радикал группы $H$, а также $N^{1}(H)=N_{G}\left(U^{1}(H)\right)$ - нормализатор группы $U^{1}$ в $G$. Далее определим индуктивно $U^{i}(H)=R_{u} N^{i-1}(H)$ и $N^{i}(H)=N_{G}\left(U^{i}(H)\right)$. Этот процесс стабилизируется начиная с некоторого $k$. Обозначим через $\mathscr{P}(H)$ подгруппу, равную $N^{k}(H)$.

ОПРЕДЕЛЕНИЕ 6 . Подгруппа $H$ группы $G$ называется правильно вложенной, если $R_{u} H \subset R_{u} G$.

Заметим, что для всех $g \in G$ выполняется равенство $\mathscr{P}\left(g H g^{-1}\right)=g \mathscr{P}(H) g^{-1}$ и что $H$ правильно вложена в $\mathscr{P}(H)$.

TеоРема 13 [1], [9]. Если $G$ - связная редуктивная группа, то группа $\mathscr{P}(H)$ является параболической подгруппой группь $G$ для любой подгруппь $H \subset G$.

ЛЕмма 5. Для любой алгебраической группы $G$ и любой подгруппы $H \subset G$ выполняется $R_{u} G \subset \mathscr{P}(H)$.

ДоКАЗАТЕЛЬСТво. Пусть $V=R_{u} \mathscr{P}(H)$ и $U=R_{u} G$. ПредПоложим, что $U \not \subset V$. Тогда по теореме Энгеля естественное действие $V$ на алгебре $\operatorname{Lie}(U /(U \cap$ $V))$ имеет ненулевой неподвижный вектор $u$. Следовательно, $u \in \operatorname{Lie}\left(R_{u} N_{G}(V)\right)$. Но это противоречит предположениям $\operatorname{Lie}(V)=\operatorname{Lie}\left(R_{u} N_{G}(V)\right)$ и $u \notin \operatorname{Lie}(V)$.

ТЕОРема 14. Для любой подгруппы $H$ связной группь $G$ подгруппа $\mathscr{P}(H)$ параболическая.

ДОКАЗАТЕЛЬСТвО. Из леммы 5 следует, что $R_{u} G \subset R_{u} \mathscr{P}(H)$.

Обозначим через $M$ группу $G / R_{u} G$, через $\psi$ - естественное отображение $G \rightarrow$ $M$, через $P$ - группу $\psi(\mathscr{P}(H))$. По построению $\operatorname{Ker} \psi=R_{u} G \subset R_{u} \mathscr{P}(H) \subset \mathscr{P}(H)$ и, значит, $P=N_{M}\left(R_{u} P\right)$. По теореме 13 группа $P$ является параболической подгруппой группы $M$, а значит, группа $\mathscr{P}(H)=\psi^{-1}(P)$ является параболической подгруппой группы $G$.

Теорема 14 легко обобщается на случай произвольной группы $G$, а именно верен следуюший результат. 
СлЕДСТВИЕ. Для любой подгруппы $H$ группы $G$ подгруппа $\mathscr{P}(H)$ является конечным расширением параболической подгруппь.

6.2. Относительные сечения и параболические подгруппы. Пусть $\alpha-$ действие группы $G$ на многообразии $X$. Обозначим через $G_{x}$ стабилизатор точки $x \in X$.

Лемма 6. Cуществует такое густое подмножество $W \subset X$, ито для любой точки $x \in W$ подгруппа $\mathscr{P}\left(G_{x}\right)$ сопряжена некоторой фиксированной nodzpynne $P_{\alpha}$.

ДокАЗАТЕЛьСтво. По теоремам 6 и 14 можно выбрать квазисечение $S$ действия $\alpha$, чтобы для всех точек $x \in S$ подгруппы $\mathscr{P}\left(G_{x}\right)$ были бы равны. Обозначим эту подгруппу через $P_{\alpha}$ и положим $W=G S$. По определению квазисечения подмножество $W$ густо в $X$.

ЛЕмма 7. Всякое действие $\alpha$ группь $G$ на многообразии $X$ допускает $P_{\alpha}$-сечение.

ДокаЗАТЕЛЬСтво. Обозначим через $S$ множество неприводимых компонент максимальной размерности многообразия $\left\{x \in X \mid \mathscr{P}\left(G_{x}\right)=P_{\alpha}\right\}$. Проверим условия относительного сечения:

1) по лемме 6 существует такое густое подмножество $W \subset X$, что $G S$ густо в $W$, а значит, $\overline{G S}=X$;

2) для любых $g \in P_{\alpha}$ и $x \in S$ имеем

$$
\mathscr{P}\left(G_{g x}\right)=\mathscr{P}\left(g G_{x} g^{-1}\right)=g \mathscr{P}\left(G_{x}\right) g^{-1}=g P_{\alpha} g^{-1}=P_{\alpha} ;
$$

3) для любых $x, y \in S$ и $g \in G$ таких, что $g x=y$, имеем

$$
P_{\alpha}=\mathscr{P}\left(G_{y}\right)=g \mathscr{P}\left(G_{x}\right) g^{-1}=g P_{\alpha} g^{-1}
$$

(это означает, что $g \in N_{G}\left(P_{\alpha}\right)=P_{\alpha}$ ).

ОПРЕДЕЛЕниЕ 7 . Действия группы $G$ на многообразии $X$ будем называть действием с правильно вложенными стабилизаторами, если для любой точки $x \in X$ и всех $i \geqslant 0$ подгруппы $N^{i}\left(G_{x}\right)$ (см. п. 6.1) правильно вложены в $G$ и, следовательно, групшы $\mathscr{P}\left(G_{x}\right)$ равны $G$.

Легко видеть, что действие групшы $P_{\alpha}$ на $P_{\alpha}$-сечении является действием с правильно вложенными стабилизаторами.

Теорема 15. Существует естественная биекция между классами бираuионально изоморфных действий группы $G$ с полем инвариантов $K$ и классами бирачионально изоморфных действий группь $P_{\alpha}$ с правильно вложенными стабилизаторами и тем же полем инвариантов.

ДокАЗАТЕльство. Обозначим через $H \subset G(\bar{K})$ стабилизатор квазисечения действия $\alpha$.

Для доказательства этой теоремы надо заметить, что $P_{\alpha}$-сечение из леммы 7 строится каноническим образом для всех действий $\beta$ группы $G$ с полем инвариантов $K$ и стабилизатором квазисечения $H$. Таким образом каждому такому действию мы поставили в соответствие действие группы $P_{\alpha}$ с теми же полем инвариантов и стабилизатором квазисечения.

Отображение в обратную сторону получается применением предложений 1 и 2. 


\section{3. Окончание доказательства теоремы 12.}

ЛЕмма 8. Пусть $\alpha-$ действие группь $G$ на многообразии X. Тогда если выполнено хотя бъ одно из следующих условий:

1) действие некоторой подгруппь $P$ на Р-сечении обладает сечением,

2) естественное действие $M: X / V$, где $G=M \wedge V$ - разложение Леви, обладает сечением,

то действие $G: X$ обладает сечением.

ДоказательСтво. 1) Легко видеть, что сечение действия группы $P$ на $P$-сечения будет и сечением действия $G: X$.

2) По теореме Розенлихта действие унипотентной групшы $V$ на $X$ допускает сечение, т. е. сушествует такое отображение $\Pi$, что $\pi \circ \Pi=\mathrm{id}_{Z}$ (см. коммутативную диаграмму):

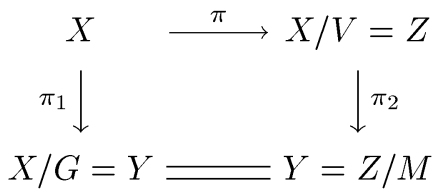

По условию существует такое отображение $\Pi_{2}$, что $\pi_{2} \circ \Pi_{2}=\mathrm{id}_{Y}$. Отображение $\Pi_{1}=\Pi \circ \Pi_{2}$ и определяет искомое сечение действия $\alpha$.

ДОКАЗАТЕЛЬСТВО ТЕОРЕМЫ 12 . Пусть вначале $\alpha$ - действие с правильно вложенными стабилизаторами.

По теореме 11 из того, что группа $G$ специальна, следует, что и ее подгруппа Леви $M$ специальна ${ }^{2}$. Значит, действие $M: X / V$ допускает сечение. По лемме 8,2 ) действие $G: X$ допускает сечение.

Из теоремы 11 и из явного описания параболических подгрупп следует, что параболическая подгруппа специальной групш специальна. Поэтому случай произвольного действия сводится к случаю действия с правильно вложенными стабилизаторами с помошью лемм 7 и 8,1$)$.

\section{§ 7. Существование относительного сечения}

7.1. Относительные сечения и разложение Леви. Пусть $G=M \curlywedge V-$ разложение Леви групшы $G$, и пусть $\alpha$ - действие $G: X$ с правильно вложенными стабилизаторами. Обозначим через $L_{x}^{0}$ подгруппу Леви стабилизатора $G_{x}$ точки $x \in X$. Аналогично обозначим через $L_{x}^{i}$ подгруппу Леви группы $N^{i}\left(G_{x}\right)$.

По теореме 6 (см. также [13]) для любого $i \geqslant 0$ существует такое густое подмногообразие $W^{i} \subset X$, что для всех $x \in W^{i}$ группы $L_{x}^{i}$ сопряжены некоторой подгруппе $L^{i} \subset M$. Без ограничения общности можно также считать, что $L=L^{0} \subset L^{1} \subset L^{2} \subset \cdots \subset L^{k}=M$.

Лемма 9. Для любого $i \geqslant 0$ действие $\alpha$ допускает $N_{M}\left(L^{i}\right) \wedge V$-сечение.

ДокАЗАТЕЛЬСТво. Легко проверить, что объединение компонент подмногообразия $S^{i}=\left\{x \in W^{i} \mid L_{x}^{i}=L^{i}\right\} \subset X$ максимальной размерности является $N_{M}\left(L^{i}\right) \curlywedge V$-сечением.

\footnotetext{
${ }^{2}$ Специальность $M$ можно доказать исходя из теоремы 3 или предложения 5 .
} 
7.2. Действия без относительных сечений. Пусть $G: X$ - некоторое действие.

ОПРЕДЕЛЕНИЕ $8 . P$-сечение действия $\alpha$ называется собственным относительным сечением, если $P \varsubsetneqq G$.

Зададимся вопросом: как описать все действия алгебраических групп $G$ на неприводимых многообразиях, не допускающие собственного относительного сечения? Полученные в этой работе результаты дают некоторый ответ на этот вопрос.

Воспользуемся обозначениями п. 7.1.

ТЕОРема 16. Для того чтобы действие $\alpha$ группы $G$ на многообразии $X$ не допускало собственного относительного сечения, необходимо выполнение следующих условий:

1) $\alpha$-действие с правильно вложсенными стабилизаторами;

2) $N_{M}\left(L^{i}\right)=M$ для всех $i$;

3) если для некоторого густого подмножества $W \subset X$ подгруппы $U^{i}\left(G_{x}\right) \subset$ $G$ сопряжены некоторой подгруппе $U \subset G$ при некотором иелом $i$, то $U=V$.

ДокАЗАТЕльство. 1) Если действие $\alpha$ не является действием с правильно вложенными стабилизаторами, то $P_{\alpha}$-сечение (лемма 7 ) будет собственным.

Дальше в доказательстве ограничимся действиями с правильно вложенными стабилизаторами.

2) Если для какого-то $i$ выполнено $N_{M}\left(L^{i}\right) \neq M$, то $N_{M}\left(L^{i}\right)<V$-сечение (лемма 9) будет собственным.

3) Пусть для всех $x$ из некоторого густого подмногообразия $W \subset X$ подгруппы $U^{i}\left(G_{x}\right) \subset G$ сопряжены некоторой подгруппе $U$ при некотором целом $i$. Объединение $S$ неприводимых компонент максимальной размерности многообразия $\{x \in$ $\left.W \mid U^{i}\left(G_{x}\right)=U\right\} \subset X$ является $N_{G}(U)$-сечением. Группа $L^{i}$ является подгруппой Леви группы $N_{G}(U)$. По предложению 4 действие $U^{2}(U): S$ допускает $L^{i}$-инвариантное сечение $S^{\prime}$. Подмногообразие $S^{\prime \prime}=U \cdot S^{\prime} \subset S \subset X$ является $L^{i} \curlywedge U$-сечением. Если $U \neq V$, то построенное $L^{i} \curlywedge U$-сечение будет собственным.

Покажем, что указанные необходимые условия не являются достаточными. А именно, отдельный случай, когда выполняются условия теоремы 16 , составляют локально свободные действия редуктивных групп. В частности, для любого такого действия нетривиальной специальной группы всегда сушествует собственное $\{e\}$-сечение (в качестве такового можно взять любое сечение).

7.3. Относительные сечения и бирациональная классификация действий. Воспользуемся обозначениями предыдущего пункта.

Рассмотрим некоторое действие $\alpha$ группы $G$ на многообразии $X$ с правильно вложенными стабилизаторами и с полем инвариантов $K$. Пусть $M \prec V$-разложение Леви группы $G$.

Обозначим через $G^{\prime}$ группу, определяемую следующим образом. Унипотентный радикал группы $G^{\prime}$ равен $V$, а подгруппа Леви равна $\cap_{i} N_{M}\left(L^{i}\right)$.

Лемма 10. Любое действие $\alpha$ допускает $G^{\prime}$-сечение. 
ДокАЗАТЕльство. Обозначим через $M^{0}$ группу $M$, а через $M^{j+1}-$ группу $N_{M^{j}}\left(L^{j}\right)$. По лемме 9 действие $G=\left(M^{0} \curlywedge V\right): X$ допускает $\left(M^{1} \prec V\right)$-сечение $X^{1}$.

Также по лемме 9 действие $M^{j} \wedge V: X^{j}$ допускает $\left(M^{j+1} \curlywedge V\right)$-сечение $X^{j+1}$.

Для продолжения доказательства нам потребуется следуюшая лемма.

ЛЕмма 11. Возьмем вложение групп $H^{2} \subset H^{1} \subset G$. Пусть действие $G: X$ допускает $H^{1}$-сечение $X^{1}$, а действие $H^{1}$ : $X^{1}$ допускает относительное $H^{2}$-сечение $X^{2}$. Тогда $X^{2}$ является $H^{2}$-сечением действия $G: X$.

ДокАЗАТЕльСТво.Проверим выполнение условий из определения относительного сечения.

1) Множество $G X^{2}=G\left(H^{1} X^{2}\right)$ густо в $X$, так как $H^{1} X^{2}$ густо в $X^{1}$, а $G X^{1}$ густо в $X$.

2) Если $g x=y$, где $g \in G$, а $x, y \in X^{2}$, то $g \in H^{1}$, так как $x, y \in X^{2} \subset X^{1}$. Но $g \in H^{2}$, так как $g \in H^{1}$ и $x, y \in X^{2}$.

Воспользовавшись теперь леммой 11 , получаем, что действие $G: X$ допускает $\left(M^{j} \wedge V\right)$-сечения для любого $j$.

В то же время для $j>0$ группа $M^{j}$ равна группе $\bigcap_{i \leqslant j} N_{M}\left(L^{i}\right)$. Так как $M^{j}=M^{j+1}$ начиная с некоторого $k$, то $M^{k}=\bigcap_{i} N_{M}\left(L^{i}\right)$ и, следовательно, $\left(M^{k} \curlywedge V\right)=G^{\prime}$.

Но, как было показано вьше, действие $G: X$ допускает $\left(M^{k} \curlywedge V\right)$-сечение.

Теперь выберем у этого $G^{\prime}$-сечения стабилизатор $H$ некоторого квазисечения.

Теорема 17. Существует естественная биекиия между классами бираиионально изоморфных действий группы $G$ с правильно вложенными стабилизаторами и с полем инвариантов $K$ и классами бирационально изоморфных действий группы $G^{\prime}$ с теми же стабилизатором квазисечения и полем инвариантов.

ДокАЗАТЕЛЬСТво. Обозначим через $H$ стабилизатор квазисечения действия $\alpha$.

Для доказательства этой теоремы надо заметить, что $G^{\prime}$-сечение из леммы 10 строится каноническим образом для всех действий $\beta$ группы $G$ с полем инвариантов $K$ и стабилизатором квазисечения $H$. Таким образом, каждому такому действию мы поставили в соответствие действие групшы $G^{\prime}$ с теми же полем инвариантов и стабилизатором квазисечения.

Отображение в обратную сторону получается применением предложений 1 и 2.

Автор выражает благодарность профессору Э.Б. Винбергу за постоянное внимание к работе.

\section{Список литературы}

1. Вейсфейлер Б. Ю. Об одном классе унипотентных подгрупп полупростых алгебраических групп // УМН. 1966. Т. 21. № 2. С. 222-223.

2. Винберг Э. Б., Онищик А. Л. Семинар по группам Ли и алгебраическим группам. М.: Наука, 1988.

3. Винберг Э. Б., Попов В. Л. Теория инвариантов // Итоги науки и техн. Соврем. пробл. матем. Фундам. направл. Т. 55. М.: ВИНИТИ, 1989. С. 137-309. 
4. Кацыло П. И. Рациональность пространств модулей гиперэллиптических кривых // Изв. АН СССР. Сер. матем. 1984. Т. 48. № 4. С. 705-710.

5. Морозов В.В. Классификация нильпотентных алгебр Ли 6-го порядка // Изв. вузов. Матем. 1958. № 4. С. 161-174.

6. Платонов В. П., Рапинчук А. С. Алгебраические группы и теория чисел. М.: Наука, 1991.

7. Серр Ж.-П. Когомологии Галуа. М.: Мир, 1968.

8. Спрингер Т.А. Линейные алгебраические группы // Итоги науки и техн. Соврем. пробл. матем. Фундам. направл. Т. 55. М.: ВИНИТИ, 1989. С. 5-136.

9. Хамфри Дж. Линейные алгебраические группы. М.: Наука, 1980.

10. Kraft H., Schwarz G. W. Reductive Group Actions with one-dimensional quotient // Publ. Math. IHES. 1992. V. 76. P. 1-97.

11. Lang S. On quasi-algebraic clouser // Ann. Math. 1952. V. 55. P. 373-390.

12. Popov V. L. Sections in Invariant Theory // The Sophus Lie Memorial Conference. Oslo, 1992. Proceedings. Scandinavian University Press, 1994. P. 315-361.

13. Richardson R. U. Deformations of Lie subgroups and the variation of isotropy subgroups // Acta Math. 1972. V. 129. № 1-2. P. 35-73.

14. Rosenlicht M. Some basic theorems on algebraic groups // Amer. J. Math. 1956. V. 178. P. 401-443.

15. Séminaire C. Chevalley. Anneaux de Chow et applications. 2nd année, IHP, 1958.

16. Serre J.-P. Cohomologie Galoisienne: Progrès et Problèms: Sémimaire BOURBAKI. 46ème, année. 1993-94. № 783.

17. Sansuc J. J. Groupe de Brauer et arithmétique des groupes algébriques linéairessur un corps de nombers // J. Reine Angew. Math. 1981. V. 327. P. 12-80.

18. Vinberg E. B. On invariants of a set of matrices // J. of Lie Theory. 1996. V. 6. P. 249-269.

Поступило в редакцию 